\title{
Aetiological bases of 46,XY disorders of sex development in the Hong Kong Chinese population
}

Angel OK Chan *, WM But, CY Lee, YY Lam, KL Ng, PY Loung, Almen Lam, CW Cheng, CC Shek, WS Wong, KF Wong, MY Wong, WY Tse

\section{A B S T R A C T}

Objective: Disorders of sex development are due to congenital defects in chromosomal, gonadal, or anatomical sex development. The objective of this study was to determine the aetiology of this group of disorders in the Hong Kong Chinese population.

Design: Case series.

Setting: Five public hospitals in Hong Kong.

Patients: Patients with 46,XY disorders of sex development under the care of paediatric endocrinologists between July 2009 and June 2011.

Main outcome measures: Measurement of serum gonadotropins, adrenal and testicular hormones, and urinary steroid profiling. Mutational analysis of genes involved in sexual differentiation by direct DNA sequencing and multiplex ligation-dependent probe amplification.

Results: Overall, 64 patients were recruited for the study. Their age at presentation ranged from birth to 17 years. The majority presented with ambiguous external genitalia including micropenis and severe hypospadias. A few presented with delayed puberty and primary amenorrhoea. Baseline and post-human chorionic gonadotropin-stimulated testosterone and dihydrotestosterone levels were not discriminatory in patients with or without $A R$ gene mutations. Of the patients, 22 had a confirmed genetic disease, with 11 having $5 \alpha$-reductase 2 deficiency, seven with androgen insensitivity syndrome, one each with cholesterol side-chain cleavage enzyme deficiency, Frasier syndrome, NR5A1-related sex reversal, and persistent Müllerian duct syndrome. are possibly the two most common causes of $46, \mathrm{XY}$ disorders of sex development in the Hong Kong Chinese population. Since hormonal findings can be unreliable, mutational analysis of the SRDSA2 and $A R$ genes should be considered the first-line tests for these patients.

\section{Hong Kong Med J 2015;21:499-510 DOI: 10.12809/hkmj144402}

\begin{abstract}
AOK Chan *\#, MD, FHKAM (Pathology)
${ }^{2}$ WM But \#, MB, BS, FHKAM (Paediatrics)

${ }^{3}$ CY Lee, MB, BS, FHKAM (Paediatrics)

${ }^{4}$ YY Lam, MB, BS, FHKAM (Paediatrics)

${ }^{5} \mathrm{KL} \mathrm{Ng}$, MB, BS, FHKAM (Paediatrics)

${ }^{6}$ PY Loung, MB, ChB, FHKAM (Paediatrics)

${ }^{5}$ A Lam, MB, ChB, FHKAM (Paediatrics)

${ }^{1} \mathrm{CW}$ Cheng, MSc

${ }^{1}$ CC Shek, MB, BS, FRCPath

${ }^{1}$ WS Wong, MB, ChB, FHKCPath

${ }^{1}$ KF Wong, MD, FHKCPath

${ }^{2}$ MY Wong, MB, ChB, FHKAM (Paediatrics)

${ }^{2}$ WY Tse, MB, BS, FHKAM (Paediatrics)
\end{abstract}

\section{Department of Pathology, Queen Elizabeth Hospital, Jordan, Hong Kong ${ }^{2}$ Department of Paediatrics, Queen Elizabeth Hospital, Jordan, Hong Kong \\ Department of Paediatrics and Adolescent Medicine, Caritas Medical Centre, Shamshuipo, Hong Kong \\ ${ }^{4}$ Department of Paediatrics and Adolescent Medicine, Kwong Wah Hospital, Yaumatei, Hong Kong \\ ${ }^{5}$ Department of Paediatrics and Adolescent Medicine, United Christian Hospital, Kwun Tong, Hong Kong \\ ${ }^{6}$ Department of Paediatrics and Adolescent Medicine, Princess Margaret Hospital, Laichikok, Hong Kong}

* Corresponding author: cok436@ha.org.hk

\# AOK Chan and WM But have equal contribution in this study.

New knowledge added by this study

- The most common likely causes of 46,XY disorders of sex development (DSD) in our local Chinese population are $5 \alpha$-reductase 2 deficiency and androgen insensitivity syndrome.

- Blood hormone testing is unreliable in differentiating between androgen insensitivity syndrome and other causes of 46,XY DSD.

- Mutational analysis of the SRD5A2 and $A R$ genes should be considered the first-line investigation in patients with 46,XY DSD.

Implications for clinical practice or policy

- When encountering patients with 46,XY DSD, $5 \alpha$-reductase 2 deficiency and androgen insensitivity syndrome should be considered early as their presence has implications for treatment and prognosis. 


\section{香港華籍人口中 46-XY單純性腺發育不全綜合徵 的病因}

\section{陳安琪、畢慧文、李靜賢、林琬瑜、吳國樑、梁寶兒、林麗}

娜、鄭俊華、石志忠、黃慧珊、黃傑輝、黃敏儀、謝詠儀

目的：性腺發育不全主要由於先天性的染色體和性腺發育異常, 或解 剖性別非典型所致。本研究旨在探討香港華籍人口中性腺發育不全的 病因。

設計：病例系列研究。

安排：香港5間公立醫院。

患者：2009年7月至2011年6月期間接受兒童內分泌科醫生診治的 46-XY單純性腺發育不全綜合徵患者。

主要結果測量 : 量度患者的血清促性腺激素、腎上腺和睪丸激素水 平, 以及尿中類固醇激素水平測試。採用DNA直接測序方法和多重連 接探針擴增術來分析基因突變。

結果：共64名患者被納入研究, 病發年齡從出生至 17 歲不等。大多數 患者的外生殖器分化模糊, 如小陰莖和嚴重的尿道下裂。少數患者的 青春期發育延遲, 並有原發性閉經。不論患者是否有 $A R$ 基因突變, 其基線和後人類絨毛膜促性腺激素刺激辠酮和雙氫睪酮水平都沒有分 別。22名患者確診有遺傳病, 其中 11 例有 $5 \alpha$ 還原酶 2 缺乏症, 7 例有 雄激素不敏感綜合徵, 另膽固醇側鏈裂解酶缺乏症、Frasier綜合徵、 與NR5A 1 基因相關的性逆轉和Müllerian管發育不全綜合徵分別各 1 例。

結論：本研究結果顯示香港華籍人口中 46-XY單純性腺發育不全綜 合徵患者最普遍的兩種病因為 $5 \alpha$ 還原酶 2 缺乏症和雄激素不敏感綜 合徵。由於激素檢測的結果未完全可靠, 應考慮為這些病人進行 $S R D 5 A 2$ 和 $A R$ 基因突變分析作為第一線檢查。

\section{Introduction}

Disorders of sex development (DSD) are defined as congenital conditions in which development of chromosomal, gonadal, or anatomical sex is atypical. ${ }^{1}$ Traditionally, diagnosis in these patients relies on extensive endocrine investigation. With advances in the understanding of the genes involved in sexual determination and differentiation, ${ }^{2}$ molecular diagnosis is playing an increasingly important role and may even overtake the role of hormonal assessment as the first-line test, with the latter being reserved for assessment of disease severity rather than diagnosis. ${ }^{3}$

One of the most common causes of 46,XY DSD in the western population is androgen insensitivity syndrome (AIS). ${ }^{4}$ Whether the same is true in our local population remains unknown. We performed a prospective multicentre study to explore the possible aetiological basis of 46,XY DSD in the Hong Kong Chinese population.

\section{Methods}

\section{Patients}

Patients who were referred to a paediatric endocrinologist for the first time or were followed up in their clinic at five public hospitals in Hong Kong between July 2009 and June 2011 were recruited for the study. Inclusion criteria were 46,XY ethnic Chinese patients who presented with incompletely virilised, ambiguous, or completely female external genitalia. Criteria that suggested DSD at birth were overt genital ambiguity, apparent female genitalia with an enlarged clitoris, posterior labial fusion, or an inguinal/labial mass, apparent male genitalia with bilateral undescended testes, micropenis, isolated perineal hypospadias, or mild hypospadias with undescended testes, and discordance between genital appearance and prenatal karyotype. ${ }^{1}$ Micropenis is defined as stretched penile length of $<2.5 \mathrm{~cm}$ based on the published norm for Chinese. ${ }^{1}$ Written informed consent was obtained from the patients and/or parents and the study was approved by the local ethics committee. None of the patients/ parents refused to participate in the study although seven refused genetic testing (Table 1).

\section{Hormone analysis}

Blood was taken from patients for electrolyte and baseline endocrine assessment and included measurement of cortisol, 17-hydroxyprogesterone (17-OHP), dehydroepiandrosterone sulfate, testosterone (T), androstenedione (A4), dihydrotestosterone (DHT), anti-Müllerian hormone (AMH), and gonadotropins. Human chorionic gonadotropin (hCG) stimulation test was performed to test for testicular Leydig cell function. The short synacthen test was also performed when indicated.

Cortisol, dehydroepiandrosterone sulfate, and gonadotropins were measured by electrochemiluminescence immunoassay (Modular Analytics E170; Roche, Mannheim, Germany); T was measured by a competitive immunoenzymatic assay (ACCESS 2; Beckman Coulter, Brea [CA], US); 17-OHP was measured by liquid chromatographytandem mass spectrometry using an in-house method; $\mathrm{AMH}$ was measured by an enzyme-linked immunosorbent assay (AMH Gen II ELISA, A73818; Beckman Coulter, Brea [CA],US);DHT was measured by radioimmunoassay (DSL9600i; Beckman Coulter, Prague, Czech Republic); A4 was measured by solid-phase competitive chemiluminescent enzymelabelled immunoassay (L2KAO2, Immulite 2000; Siemens, Tarrytown [NY], US). Male reference intervals were considered the most appropriate for data interpretation in this study.

\section{Urinary steroid profiling}

Spot urine from patients under 3 months of age and 24-hour urine from those at or older than 3 months of age were processed for steroid profiling as described previously. ${ }^{5}$ 


\section{Molecular analysis}

DNA was extracted from peripheral whole blood using a QIAamp DNA blood kit (Qiagen, Hilden, Germany). Polymerase chain reaction and direct DNA sequencing were performed on targeted genes when suggested by the clinical and hormonal findings. Otherwise all patients had their $A R$ (androgen receptor) and NR5A1 (steroidogenic factor 1) genes sequenced. Those patients with negative genetic findings were subjected to multiplex ligationdependent probe amplification (MLPA) analysis (P185 Intersex probemix; P074 Androgen Receptor probemix and P334 Gonadal probemix; MRCHolland) to test for gross deletion or gene duplication. The results were analysed by Coffalyser.Net. Family genetic studies were performed when mutation(s) were identified in the index patients.

\section{In-silico analysis for novel missense mutations}

The functional effect of novel missense mutations detected was tested by online in-silico analysis software SIFT, PolyPhen2, and Align GVGD.

\section{Results}

Overall, 64 patients (53 male, 11 phenotypic female), including 14 new patients, with 46,XY DSD were recruited into the study. The clinical and hormonal findings of individual patients are listed in Table 1. A genetic diagnosis was made in 10 patients prior to the study. Other major structural abnormalities were evident in eight (Table 2). Their age at presentation ranged from birth to 17 years. Five $(8 \%)$ were born prematurely (24-35 weeks) and nine (14\%) with low birth weight $(0.59-2.32 \mathrm{~kg})$. All had non-consanguineous parents. A family history of sexual ambiguity was present in six. Overall, 61 (95\%) presented with ambiguous external genitalia including 15 with isolated micropenis, eight with isolated severe hypospadias, and one with discordance between the prenatal karyotype and the postnatal phenotype. Three presented after birth, one each with inguinal hernia, delayed puberty, and primary amenorrhoea.

Regarding the hormonal findings, Figure 1 shows the baseline and post-hCG-stimulated $\mathrm{T}$ and DHT levels in patients with mutations detected in the $A R$ gene and those without, where the results overlapped between the two groups. Eight patients (patients 13, 19, 21, 30, 31, 40, 49, and 56) underwent short synacthen test and with the exception of patient 19, all had an adequate cortisol response ( $>550 \mathrm{nmol} / \mathrm{L}$ ). Patient 27 had a relatively low T/A4 ratio before and after hCG stimulation but sequencing revealed no mutation in his $H S D 17 B 3$ (17 $\beta$-hydroxysteroid dehydrogenase III) gene. All other patients had unremarkable $\mathrm{T}$ and $\mathrm{A} 4$ levels, as well as T/A4 ratio.

Eleven patients had characteristically low $5 \alpha$ - to $5 \beta$-reduced steroid metabolite ratios in their urine, compatible with the diagnosis of $5 \alpha-$ reductase 2 deficiency (5ARD). This was also confirmed by mutational analysis of the SRD $5 A 2$ (steroid $5 \alpha$-reductase 2 ) gene. All other patients had unremarkable urinary steroid metabolite pattern.

Overall, 22 (39\%) patients had a confirmed genetic diagnosis (Table 3). The most common diagnoses in our cohort were 5ARD $(n=11)$ and AIS $(n=7)$. Other genetic diagnoses included cholesterol side-chain cleavage enzyme deficiency $(n=1)$, Frasier syndrome $(\mathrm{n}=1)$, NR5A1-related sex reversal $(\mathrm{n}=1)$, and persistent Müllerian duct syndrome (PMDS; $\mathrm{n}=1$ ). The clinical and laboratory findings of patients 19 and 20 have been reported previously. ${ }^{6,7}$ Patients 12 and 15 had de-novo mutations in the $A R$ gene and were in mosaic pattern. Patient 21 had a novel missense variant p.Ala260Val detected in his NR5A1 gene. His AMH level was not low, contrary to some of the previously reported cases. ${ }^{8}$ There was also a clinically significant rise in $\mathrm{T}$ level after hCG stimulation. Short synacthen test demonstrated an adequate cortisol response (baseline: $720 \mathrm{nmol} / \mathrm{L}$; post-adrenocorticotropin hormone: $822 \mathrm{nmol} / \mathrm{L}$ ). His father also carried the same heterozygous mutation although he denied any symptoms of DSD. This novel genetic variant was not detected in 100 normal Chinese subjects (control). Patient 22 had bilateral undescended testes. He underwent orchidopexy at the age of 1 year during which the presence of Müllerian duct structures was suspected. Further workup including pelvic ultrasound revealed Müllerian duct structures and extremely low AMH level. The diagnosis of PMDS was confirmed by the presence of three heterozygous novel missense variants in the $A M H$ gene (Tables 3 and 4).

Six novel genetic variants were identified in the $A M H, A R$, and NR5A1 genes (Fig 2). At least two of the three in-silico analysis programmes predicted the variants to be pathogenic (Table 4). Multiple sequence alignment showed that the amino acids of concern were highly conserved across different animal species. All these findings support the pathogenic nature of these variants accounting for the patients' phenotypes.

Eleven patients were reared as girls because of severe under-virilisation at birth, including three with 5ARD, three with AIS, and one with Frasier syndrome. The underlying genetic causes in the remaining four patients were undetermined. The longest follow-up period was 27 years. None of them has requested change of gender to date. Five patients (patients 2, 4, 7, 12, and 15) exhibited 'tomboy-like' behaviour during childhood and required counselling by a clinical psychologist while two males (patients 17 and 47) requested exogenous $\mathrm{T}$ 
to augment penile growth after puberty. Patient 20 clinical management can be formulated, bearing in developed germinoma in her dysgenetic gonad with mind the best interests of the child. Initial workup no recurrence after surgery.

\section{Discussion}

$46, \mathrm{XY}$ DSD is a heterogeneous condition caused by a wide spectrum of disorders. Making an accurate diagnosis is difficult but important for emergency medical treatment as some DSDs are associated with life-threatening Addisonian crisis. In addition, the diagnosis is essential so that relevant information and counselling can be provided to parents and includes a detailed antenatal and postnatal history, physical examination, karyotyping, and hormonal assays. This will guide further workup such as imaging and genetic analysis. Nonetheless, there are often limitations to hormonal studies as illustrated in the present series. The non-distinct pattern of $\mathrm{T}$ and DHT at baseline and following hCG stimulation in $A R$ mutation-positive and -negative patients suggest the need to reconsider our laboratory diagnostic algorithm for AIS.

TABLE I. The clinical and hormonal findings of 64 patients with 46,XY disorders of sex development recruited in this study. Those baseline hormonal results below the age- and gender-specific reference limits are underlined, those above are in bold

\begin{tabular}{|c|c|c|c|c|c|c|c|c|c|}
\hline \multirow{2}{*}{$\begin{array}{l}\text { Pa- } \\
\text { tient } \\
\text { No. }\end{array}$} & \multirow{2}{*}{$\begin{array}{l}\text { Sex of } \\
\text { rearing }\end{array}$} & \multirow[t]{2}{*}{ Presentation } & \multirow{2}{*}{$\begin{array}{c}\text { Age at } \\
\text { presen- } \\
\text { tation }\end{array}$} & \multirow{2}{*}{$\begin{array}{c}\text { Age at } \\
\text { recruitment } \\
\text { and hormonal } \\
\text { evaluation }\end{array}$} & \multirow{2}{*}{$\begin{array}{l}\text { Other } \\
\text { proced- } \\
\text { ures }\end{array}$} & \multicolumn{4}{|c|}{ Baseline } \\
\hline & & & & & & LH (IU/L) & FSH (IU/L) & $\begin{array}{l}\text { Cortisol } \\
\text { (nmol/L) }\end{array}$ & $\begin{array}{l}\text { DHEAS } \\
\text { ( } \mu \mathrm{mol} / \mathrm{L})\end{array}$ \\
\hline \multicolumn{10}{|c|}{ 5ARD } \\
\hline 1 & $M$ & AEG & At birth & 3 Years & $\mathrm{O}$ & $<0.2$ & 0.6 & 268 & $\leq 0.4$ \\
\hline 2 & $\mathrm{~F}$ & AEG & At birth & 26 Years & $\mathrm{BG}+\mathrm{V}$ & $<0.2$ & 1.7 & - & - \\
\hline 3 & M & Micropenis & At birth & 8 Years & - & $<0.2$ (At 1 year) & 0.8 (At 1 year) & 163 & - \\
\hline 4 & $\mathrm{~F}$ & AEG & At birth & 20 Years & $B G+V$ & - & - & - & - \\
\hline 5 & $\mathrm{M}$ & Micropenis & At birth & 7 Years & - & 2.6 (On 18 days) & 1.3 & 167 & - \\
\hline 6 & $M$ & Hypospadia, micropenis & At birth & 4 Years & - & 2.7 & 2.4 & 68 & - \\
\hline $7^{*}$ & $\mathrm{~F}$ & AEG & 2 Months & 26 Years & $\mathrm{BG}+\mathrm{V}$ & - & - & - & - \\
\hline 8 & $\mathrm{M}$ & Micropenis & 1 Year & 2 Years & - & - & - & - & - \\
\hline 9 & $M$ & Micropenis & 2 Years & 15 Years & - & 4.3 & 4.0 & 207 & 2.9 \\
\hline 10 & $M$ & Micropenis & 2 Years & 5 Years & - & 0.5 & 1.0 & - & - \\
\hline 11 & $\mathrm{M}$ & Micropenis & 17 Years & 17 Years & - & 6.6 & 7.1 & - & - \\
\hline \multicolumn{10}{|c|}{ Androgen insensitivity syndrome } \\
\hline $12^{\star}$ & $\mathrm{F}$ & AEG & At birth & 27 Years & $\mathrm{BG}+\mathrm{V}$ & - & - & 152 & $\underline{3.6}$ \\
\hline 13 & $M$ & AEG & At birth & 5 Months & $\mathrm{R}$ & 2.3 & 1.5 & 623 & - \\
\hline 14 & M & AEG & At birth & 10 Years & $\mathrm{R}$ & $<0.2$ & 2.5 & 273 & 2.3 \\
\hline $15^{\star}$ & $\mathrm{F}$ & AEG & At birth & 14 Years & $B G$ & - & - & 73 & 5.8 \\
\hline 16 & $M$ & AEG & At birth & 15 Days & $\mathrm{R}$ & 22.7 & 11.5 & 63 & $<0.4$ \\
\hline 17 & M & AEG & 1 Month & 16 Years & $\mathrm{R}$ & 14.6 & 5.9 & 347 & 8.0 \\
\hline 18 & $\mathrm{~F}$ & Primary amenorrhoea & 15 Years & 16 Years & $B G$ & 16.2 & 11.6 & - & - \\
\hline \multicolumn{10}{|c|}{ Cholesterol side-chain cleavage enzyme deficiency } \\
\hline 19 & $M$ & AEG & At birth & 2 Years & $\mathrm{O}$ & 7.3 & 27.3 & 54 & $\leq 0.4$ \\
\hline \multicolumn{10}{|c|}{ Frasier syndrome } \\
\hline $20^{\star}$ & $\mathrm{F}$ & Delayed puberty & 15 Years & 24 Years & $B G$ & 54.7 & 181 & - & - \\
\hline \multicolumn{10}{|c|}{ NR5A1-related sex reversal } \\
\hline 21 & $M$ & $\mathrm{AEG}$ & At birth & 5 Years & $\mathrm{R}$ & 0.8 & 1.2 & 158 & $\leq 0.4$ \\
\hline \multicolumn{10}{|c|}{ Persistent Müllerian duct syndrome } \\
\hline 22 & $\mathrm{M}$ & Bilateral undescended testes & At birth & 10 Years & Bilateral O & $<0.2$ & 1.1 & 173 & 2.4 \\
\hline
\end{tabular}

Abbreviations: - = test not done; A4 = androstenedione; $5 \mathrm{ARD}=5 \alpha$-reductase 2 deficiency; $\mathrm{AEG}=$ anophthalmia-oesophageal-genital; $\mathrm{AMH}=$ anti-Müllerian hormone; BG = bilateral gonadectomy; DHEAS = dehydroepiandrosterone sulfate; DHT = dihydrotestosterone; FSH = follicle-stimulating hormone; $\mathrm{hCG}=$ human chorionic gonadotropin; $\mathrm{LH}=$ luteinising hormone; $\mathrm{N}=$ normal; $\mathrm{O}=$ orchidopexy; 17 -OHP = 17-hydroxyprogesterone; $\mathrm{R}=$ repair; $\mathrm{T}=$ testosterone; USP = urinary steroid profiling; $V=$ vaginoplasty

* Blood taken after gonadectomy 
Androgen insensitivity syndrome is reported an adult, although we have not received any such to be the most common cause of 46,XY DSD in a few request from our cohort. Exposure to androgen ethnic groups, ${ }^{9-11}$ while 5ARD, which is believed to during the antenatal, postnatal, and pubertal period be rare, was also a major aetiology in our cohort. It may masculinise the brain and influence gender is important to differentiate between 5ARD and AIS identity. ${ }^{13}$ It was found that 5ARD is easy to diagnose as soon as possible so that patients with 5ARD can by its characteristic urinary steroid excretion be raised as boys whenever practical. ${ }^{12}$ The penile pattern and its high mutational detection rate in the growth of patients with 5ARD can be promoted by $S R D 5 A 2$ gene. ${ }^{14}$ Of the 11 patients with 5 ARD, eight topical DHT treatment and spontaneous virilisation harboured the missense mutation p.Arg227Gln in may occur during puberty. Most of these patients their SRDSA2 gene, a useful fact to enable screening who are reared as girls during childhood identify for this mutation before proceeding to sequencing themselves as male and change their gender as of the whole gene. Unfortunately, patients have

\begin{tabular}{|c|c|c|c|c|c|c|c|c|c|c|c|}
\hline \multirow[b]{2}{*}{$\begin{array}{l}\text { 17-OHP } \\
\text { (nmol/L) }\end{array}$} & \multicolumn{5}{|c|}{ Baseline } & \multicolumn{5}{|c|}{ Post-hCG stimulation } & \multirow[t]{2}{*}{ USP } \\
\hline & T (nmol/L) & $\begin{array}{c}\text { AMH } \\
\text { (pmol/L) }\end{array}$ & $\begin{array}{c}\text { DHT } \\
\text { (pmol/L) }\end{array}$ & $\begin{array}{c}\mathrm{A4} \\
\text { (nmol/L) }\end{array}$ & T/A4 ratio & T (nmol/L) & $\begin{array}{c}\text { AMH } \\
\text { (pmol/L) }\end{array}$ & $\begin{array}{c}\text { DHT } \\
\text { (pmol/L) }\end{array}$ & $\begin{array}{c}\text { A4 } \\
\text { (nmol/L) }\end{array}$ & $\begin{array}{l}\text { T/A4 } \\
\text { ratio }\end{array}$ & \\
\hline 0.5 & $<0.4$ & $>100$ & $<13.8$ & $<1.0$ & - & 8.1 & $>100$ & 206 & $<1.0$ & - & 5ARD \\
\hline- & 4.3 (At 2 months) & - & - & - & - & - & - & - & - & - & 5ARD \\
\hline- & $<0.4$ (At 1 year) & - & - & - & - & 14.1 (At 1 year) & - & - & - & - & 5ARD \\
\hline- & - & - & - & - & - & - & - & - & - & - & 5ARD \\
\hline- & 6.8 & - & - & - & - & - & - & - & - & - & 5ARD \\
\hline- & 3.0 & - & - & - & - & 10.0 & - & - & - & - & 5ARD \\
\hline- & $<0.4$ & - & - & - & - & 7.4 & - & - & - & - & 5ARD \\
\hline- & - & - & - & - & - & - & - & - & - & - & 5ARD \\
\hline 2.4 & 15.7 & 51.8 & $\underline{236}$ & 6.2 & 2.53 & - & - & - & - & - & 5ARD \\
\hline- & $<0.4$ & - & - & - & - & 5.5 & - & - & - & - & 5ARD \\
\hline- & 14.3 & - & - & - & - & - & - & - & - & - & 5ARD \\
\hline$<0.5$ & - & - & $\underline{88.9}$ & - & - & - & - & - & - & - & $\mathrm{N}$ \\
\hline 1.4 & 1.7 & $>100$ & 299 & 2.6 & 0.66 & 28.3 & $>100$ & 2601 & 4.6 & 6.13 & $\mathrm{~N}$ \\
\hline 1.3 & $<0.4$ & $>100$ & 19.0 & 2.6 & - & $<0.4$ & $>100$ & 116 & 2.7 & - & $\mathrm{N}$ \\
\hline$<0.5$ & $<0.4$ & $<1.3$ & $\underline{29.4}$ & 3.1 & - & - & - & - & - & - & $N$ \\
\hline- & 6.9 & $>100$ & 2392 & 7.4 & 0.93 & 11.2 & $>100$ & 4448 & 5.6 & 2.00 & - \\
\hline 3.3 & 22.4 & 80.5 & 2115 & 4.2 & 5.33 & - & - & - & - & - & $N$ \\
\hline- & 41.2 & $>100$ & 4405 & 9.8 & 4.20 & - & - & - & - & - & $\mathrm{N}$ \\
\hline 0.9 & $<0.4$ & - & - & - & - & $<0.4$ & - & - & - & - & $\mathrm{N}$ \\
\hline- & $\leq 0.4$ & - & - & - & - & - & - & - & - & - & - \\
\hline 0.9 & $<0.4$ & $>100$ & 14.9 & 1.1 & 0.00 & 6.2 & $>100$ & 1526 & 1.7 & 3.65 & $\mathrm{~N}$ \\
\hline 0.8 & $<0.4$ & $<1.3$ & 21.0 & 1.3 & - & 4.2 & $<1.3$ & 1017 & 1.6 & 2.63 & - \\
\hline
\end{tabular}


TABLE I. (cont'd)

\begin{tabular}{|c|c|c|c|c|c|c|c|c|c|}
\hline \multirow{2}{*}{$\begin{array}{l}\text { Pa- } \\
\text { tient } \\
\text { No. }\end{array}$} & \multirow{2}{*}{$\begin{array}{l}\text { Sex of } \\
\text { rearing }\end{array}$} & \multirow[t]{2}{*}{ Presentation } & \multirow{2}{*}{$\begin{array}{l}\text { Age at } \\
\text { presen- } \\
\text { tation }\end{array}$} & \multirow{2}{*}{$\begin{array}{c}\text { Age at } \\
\text { recruitment } \\
\text { and hormonal } \\
\text { evaluation }\end{array}$} & \multirow{2}{*}{$\begin{array}{l}\text { Other } \\
\text { proced- } \\
\text { ures }\end{array}$} & \multicolumn{4}{|c|}{ Baseline } \\
\hline & & & & & & LH (IU/L) & FSH (IU/L) & $\begin{array}{l}\text { Cortisol } \\
\text { (nmol/L) }\end{array}$ & $\begin{array}{l}\text { DHEAS } \\
(\mu \mathrm{mol} / \mathrm{L})\end{array}$ \\
\hline \multicolumn{10}{|c|}{ Not confirmed genetically } \\
\hline 23 & M & $A E G$ & At birth & At birth & - & 7.3 & 2.6 & 449 & 4.6 \\
\hline 24 & M & AEG & At birth & At birth & - & - & - & - & - \\
\hline 25 & $\mathrm{~F}$ & AEG, small gonads & At birth & At birth & - & 0.4 & 0.9 & 162 & 0.8 \\
\hline 26 & M & Hypospadia & At birth & At birth & - & 0.2 & 2.2 & 100 & 0.8 \\
\hline 27 & M & Micropenis & At birth & At birth & $\mathrm{O}$ & $<0.2$ & 0.3 & 311 & 0.9 \\
\hline 28 & M & Hypospadia, bifid scrotum & At birth & At birth & - & 5.5 & 1.9 & 636 & 3.9 \\
\hline 29 & M & AEG & At birth & 5 Days & $\mathrm{O}$ & 1.5 & 1.9 & 364 & 1.6 \\
\hline 30 & M & $\begin{array}{c}\text { Micropenis, bilateral undescended } \\
\text { testes }\end{array}$ & At birth & 2 Months & - & $<0.2$ & 0.4 & 236 & 1.8 \\
\hline 31 & M & Micropenis, epispadia & At birth & 3 Years & $\mathrm{R}$ & 1.0 & 0.6 & 287 & - \\
\hline 32 & M & Hypospadia & At birth & 3 Years & $\mathrm{R}$ & $<0.2$ & 1.8 & 301 & - \\
\hline 33 & M & Hypospadia & At birth & 4 Years & $\mathrm{R}$ & $<0.2$ & 3.2 & 307 & $\leq 0.4$ \\
\hline 34 & M & AEG & At birth & 6 Years & $\mathrm{R}$ & $<0.2$ & 0.3 & 364 & 2.9 \\
\hline 35 & M & AEG & At birth & 6 Years & $\mathrm{R}$ & $<0.2$ & $<0.2$ & 217 & - \\
\hline 36 & M & Micropenis, left undescended testis & At birth & 7 Years & Left $O$ & 0.6 & 0.9 & 788 & - \\
\hline 37 & M & $A E G$ & At birth & 8 Years & $\mathrm{R}$ & - & - & - & - \\
\hline 38 & M & AEG & At birth & 9 Years & $\mathrm{R}$ & 1.2 & 4.0 & 348 & 4.1 \\
\hline 39 & M & AEG & At birth & 9 Years & $\mathrm{R}$ & 0.3 & 2.0 & 121 & 1.6 \\
\hline 40 & M & Micropenis, hypospadia & At birth & 10 Years & Left $\mathrm{O}+\mathrm{R}$ & 0.5 & 2.9 & 78 & 2.8 \\
\hline 41 & M & AEG & At birth & 10 Years & $\mathrm{R}$ & 2.0 & 4.4 & 172 & 3.8 \\
\hline 42 & $\mathrm{~F}$ & $\begin{array}{l}\text { Amniocentesis } 46, \mathrm{XY} \text {, phenotype } \\
\text { at birth normal female external } \\
\text { genitalia }\end{array}$ & At birth & 10 Years & $B G$ & 0.7 & 4.9 & 157 & 1.7 \\
\hline 43 & M & Micropenis & At birth & 11 Years & - & 0.7 & 2.1 & 146 & 5.5 \\
\hline 44 & M & AEG & At birth & 12 Years & $\mathrm{R}$ & 2.3 & 4.7 & 197 & - \\
\hline 45 & M & $A E G$ & At birth & 15 Years & $\mathrm{R}$ & 5.8 & 5.1 & 266 & 5.5 \\
\hline 46 & M & AEG & At birth & 19 Years & $\mathrm{R}$ & 5.0 & 8.4 & 352 & - \\
\hline 47 & M & $A E G$ & At birth & 23 Years & $\mathrm{R}$ & 27.3 & 13.3 & 370 & $\underline{5.8}$ \\
\hline 48 & M & Micropenis & 1 Month & 6 Months & - & 0.5 & 3.3 & 205 & $\leq 0.4$ \\
\hline 49 & M & Micropenis & 1 Month & 1 Year & - & $<0.2$ & 0.9 & 677 & $\underline{0.4}$ \\
\hline $50^{\star}$ & $\mathrm{F}$ & $\begin{array}{l}\text { Inguinal gonads with normal female } \\
\text { external genitalia }\end{array}$ & 1 Year & 23 Years & $B G$ & - & - & 164 & $\underline{0.8}$ \\
\hline 51 & M & Hypospadia & 2 Years & 13 Years & $\mathrm{R}$ & 1.8 & 2.4 & 151 & 2.8 \\
\hline 52 & M & Bilateral undescended small testis & 5 Years & 19 Years & Bilateral O & 32.6 & 70.7 & 219 & 6.1 \\
\hline 53 & M & Hypospadia & 5 Years & 13 Years & $\mathrm{R}$ & 3.7 & 7.3 & 193 & 4.7 \\
\hline 54 & M & Micropenis & 6 Years & 8 Years & - & $<0.2$ & 0.6 & 796 & $\underline{0.5}$ \\
\hline 55 & M & Hypospadia & 7 Years & 11 Years & $\mathrm{R}$ & 0.4 & 2.9 & - & - \\
\hline 56 & M & Micropenis & 9 Years & 9 Years & - & 0.7 & 3.5 & 159 & - \\
\hline 57 & M & Micropenis & 17 Years & 17 Years & - & 1.4 & 2.2 & 166 & - \\
\hline \multicolumn{10}{|c|}{ Not consented for genetic testing } \\
\hline 58 & M & Hypospadia & At birth & At birth & - & 0.5 & 1.2 & 365 & 1.2 \\
\hline 59 & M & Hypospadia & At birth & At birth & $\mathrm{R}$ & 1.0 & 1.7 & 464 & 2.6 \\
\hline 60 & $\mathrm{~F}$ & $A E G$, inguinal gonads & At birth & 2 Months & $B G$ & $<0.2$ & 2.4 & 272 & 0.9 \\
\hline 61 & M & $A E G$ & At birth & 5 Years & $\mathrm{R}$ & $<0.2$ & 1.2 & 91 & $\underline{0.4}$ \\
\hline 62 & M & Micropenis & 1 Year & 1 Year & - & - & - & - & - \\
\hline 63 & M & Micropenis, small gonads & 11 Years & 11 Years & - & $<0.2$ & 2.3 & - & - \\
\hline 64 & M & Micropenis & 11 Years & 12 Years & - & 2.4 & 2.4 & - & 0.9 \\
\hline
\end{tabular}




\begin{tabular}{|c|c|c|c|c|c|c|c|c|c|c|c|}
\hline \multirow[b]{2}{*}{$\begin{array}{l}\text { 17-OHP } \\
\text { (nmol/L) }\end{array}$} & \multicolumn{5}{|c|}{ Baseline } & \multicolumn{5}{|c|}{ Post-hCG stimulation } & \multirow[t]{2}{*}{ USP } \\
\hline & $\mathrm{T}$ (nmol/L) & $\underset{\text { (pmol/L) }}{\text { AMH }}$ & $\begin{array}{c}\text { DHT } \\
\text { (pmol/L) }\end{array}$ & $\begin{array}{c}\mathrm{A} 4 \\
\text { (nmol/L) }\end{array}$ & T/A4 ratio & $\mathbf{T}$ (nmol/L) & $\underset{\text { (pmol/L) }}{\text { AMH }}$ & $\begin{array}{c}\text { DHT } \\
\text { (pmol/L) }\end{array}$ & $\begin{array}{c}\mathrm{A4} \\
\text { (nmol/L) }\end{array}$ & $\begin{array}{l}\text { T/A4 } \\
\text { ratio }\end{array}$ & \\
\hline 6.6 & 6.6 & $>100$ & 2132 & 33.1 & 0.20 & - & - & - & - & & - \\
\hline- & 1.5 & $>100$ & 237 & 4.4 & 0.34 & 8.4 & $>100$ & 2277 & 5.2 & 1.62 & - \\
\hline 1.3 & $\leq 0.4$ & $>100$ & $\underline{35.9}$ & 15.9 & & $<0.4$ & $>100$ & 31.8 & 7.1 & - & $\mathrm{N}$ \\
\hline 0.9 & 1.3 & - & - & - & - & 6.5 & $>100$ & 36.7 & 13.9 & 0.47 & $\mathrm{~N}$ \\
\hline 0.8 & 1.1 & $>100$ & $\underline{14.4}$ & 15.0 & 0.07 & 1.6 & $>100$ & 115 & 19.3 & 0.08 & $\mathrm{~N}$ \\
\hline 4.5 & 3.5 & $>100$ & 571 & 32.4 & 0.11 & 9.0 & $>100$ & 2983 & 26.9 & 0.33 & $\mathrm{~N}$ \\
\hline 2.1 & 7.2 & $>100$ & 1543 & 14.0 & 0.51 & 12.8 & $>100$ & 3267 & - & - & $N$ \\
\hline 1.3 & $<0.4$ & $<1.3$ & 24.3 & 1.9 & - & $<0.4$ & $<1.3$ & 33.7 & 1.5 & - & $\mathrm{N}$ \\
\hline 4.8 & $<0.4$ & $>100$ & $<13.8$ & $<1.0$ & - & 11.5 & $>100$ & 1342 & $<1.0$ & - & $\mathrm{N}$ \\
\hline$<0.5$ & $<0.4$ & $>100$ & $<13.8$ & $<1.0$ & - & 9.2 & $>100$ & 362 & $<1.0$ & - & $\mathrm{N}$ \\
\hline 1.7 & 0.7 & $>100$ & $<13.8$ & $<1.0$ & - & 7.4 & $>100$ & 870 & 1.3 & 5.65 & $N$ \\
\hline 2.0 & $<0.4$ & $>100$ & - & - & - & 2.8 & $>100$ & 397 & 3.0 & 0.93 & $\mathrm{~N}$ \\
\hline 0.5 & $<0.4$ & $>100$ & 30.5 & 1.7 & 0.00 & 2.6 & $>100$ & - & 2.9 & 0.90 & $\mathrm{~N}$ \\
\hline 3.0 & 0.5 & $>100$ & 24.1 & 4.6 & 0.11 & 2.7 & $>100$ & 247 & 4.9 & 0.55 & $\mathrm{~N}$ \\
\hline- & - & $>100$ & 16.6 & 1.1 & - & 2.8 & $>100$ & 337 & 2.2 & 1.30 & $\mathrm{~N}$ \\
\hline 2.6 & 2.7 & 37.5 & 226 & 5.1 & 0.53 & 24.2 & 33.5 & 2542 & 9.4 & 2.57 & $\mathrm{~N}$ \\
\hline 0.7 & 0.4 & $>100$ & 17.6 & 2.4 & 0.17 & 6.9 & $>100$ & 264 & 1.8 & 3.83 & $\mathrm{~N}$ \\
\hline 1.1 & $<0.4$ & 12.4 & 24.6 & 2.6 & - & 12.4 & 11.6 & 234 & 4.3 & 2.88 & $\mathrm{~N}$ \\
\hline 0.5 & 0.4 & $>100$ & 87.8 & 1.3 & 0.31 & 5.5 & $>100$ & 1198 & 2.0 & 2.75 & $\mathrm{~N}$ \\
\hline 0.6 & 2.0 (On 9 days) & - & - & 1.1 & - & - & - & - & - & - & $\mathrm{N}$ \\
\hline$<0.5$ & 1.2 & $>100$ & 88.4 & 3.6 & 0.33 & 18.3 & $>100$ & 1446 & 3.9 & 4.67 & $\mathrm{~N}$ \\
\hline 1.6 & 4.1 & $>100$ & 684 & 3.1 & 1.30 & - & - & - & - & - & $\mathrm{N}$ \\
\hline 1.7 & 11.4 & 70.7 & 1220 & 6.6 & 1.73 & - & - & - & - & - & $\mathrm{N}$ \\
\hline 3.7 & 11.1 & 16.3 & 1493 & 8.6 & 1.29 & - & - & - & - & - & $\mathrm{N}$ \\
\hline 5.4 & 32.7 & 23 & 4368 & 9.0 & 3.63 & - & - & - & - & - & $\mathrm{N}$ \\
\hline 3.9 & 0.2 & $>100$ & 45.1 & $<1.0$ & - & 17.3 & $>100$ & 4899 & 3.6 & 4.79 & $\mathrm{~N}$ \\
\hline 5.7 & $<0.4$ & $>100$ & $<13.8$ & $<1.0$ & - & 8.5 & $>100$ & 668 & 1.7 & 5.12 & $\mathrm{~N}$ \\
\hline$<0.5$ & $<0.4$ & $<1.3$ & 139 & $\leq 1.0$ & - & - & - & - & - & - & $\mathrm{N}$ \\
\hline 1.6 & 13.8 & 61.3 & 1261 & 2.8 & 4.98 & - & - & - & - & - & - \\
\hline 0.5 & $\underline{3.7}$ & $<1.3$ & 385 & 3.3 & 1.12 & - & - & - & - & - & $\mathrm{N}$ \\
\hline 2.5 & 15.6 & 14.7 & 2633 & 6.0 & 2.62 & - & - & - & - & - & - \\
\hline 5.1 & $<0.4$ & $>100$ & 14.8 & 1.8 & - & 6.0 & $>100$ & 335 & 2.1 & 2.84 & $\mathrm{~N}$ \\
\hline- & $<0.4$ & $>100$ & 40.6 & 3.3 & - & 2.8 & $>100$ & 213 & 1.9 & 1.47 & $\mathrm{~N}$ \\
\hline$<0.5$ & $<0.4$ & $>100$ & 25.1 & $<1.0$ & - & 6.4 & $>100$ & 577 & 2.9 & 2.21 & $\mathrm{~N}$ \\
\hline 0.6 & 2.2 & 91.3 & $\underline{91.9}$ & $\underline{1.5}$ & 1.48 & 19.5 & - & - & - & - & $\mathrm{N}$ \\
\hline 1.2 & 2.5 & $>100$ & $\underline{79.3}$ & 15.6 & 0.16 & 9.7 & - & - & - & - & $\mathrm{N}$ \\
\hline 3.9 & 14.8 & - & 624 & - & - & 38.2 & - & - & - & - & $\mathrm{N}$ \\
\hline 1.3 & $<0.4$ & $>100$ & $\underline{66.2}$ & 2.9 & - & 25.1 & $>100$ & 4413 & 5.5 & 4.60 & $\mathrm{~N}$ \\
\hline$<0.5$ & $<0.4$ & - & - & - & - & 4.9 & - & - & - & - & $\mathrm{N}$ \\
\hline- & $<0.4$ & $>100$ & $<13.8$ & $<1.0$ & - & 6.7 & $>100$ & 873 & $<1.0$ & - & $N$ \\
\hline- & $<0.4$ & $>100$ & 36.4 & 3.5 & - & 1.6 & $>100$ & 83.3 & 2.8 & 0.58 & $N$ \\
\hline 4.7 & 2.0 & - & - & - & - & 13.3 & - & - & - & - & $N$ \\
\hline
\end{tabular}


TABLE 2. Other structural abnormalities detected in eight of the patients in this study

\begin{tabular}{ll}
\hline $\begin{array}{l}\text { Patient } \\
\text { No. }\end{array}$ & Structural abnormalities \\
\hline 4 & Neuroblastoma \\
\hline 13 & Horseshoe kidney \\
27 & Cleft palate \\
\hline 30 & Right renal agenesis \\
\hline 41 & Small patent ductus arteriosus with occlusion \\
47 & Imperforated anus \\
\hline 59 & Patent ductus arteriosus, ventricular septal defect \\
61 & Right bronchogenic cyst \\
\hline
\end{tabular}

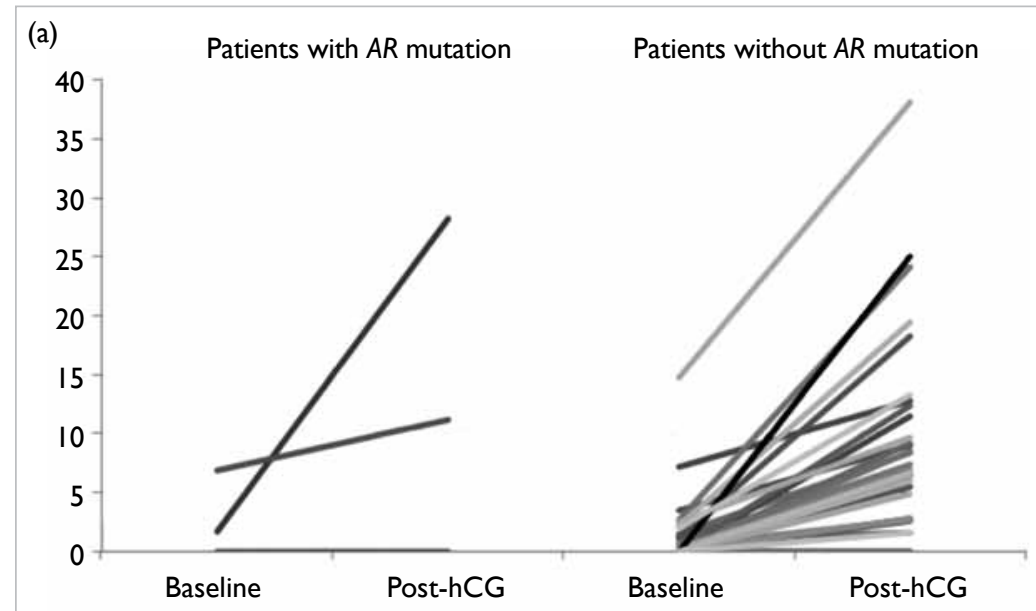

(b)

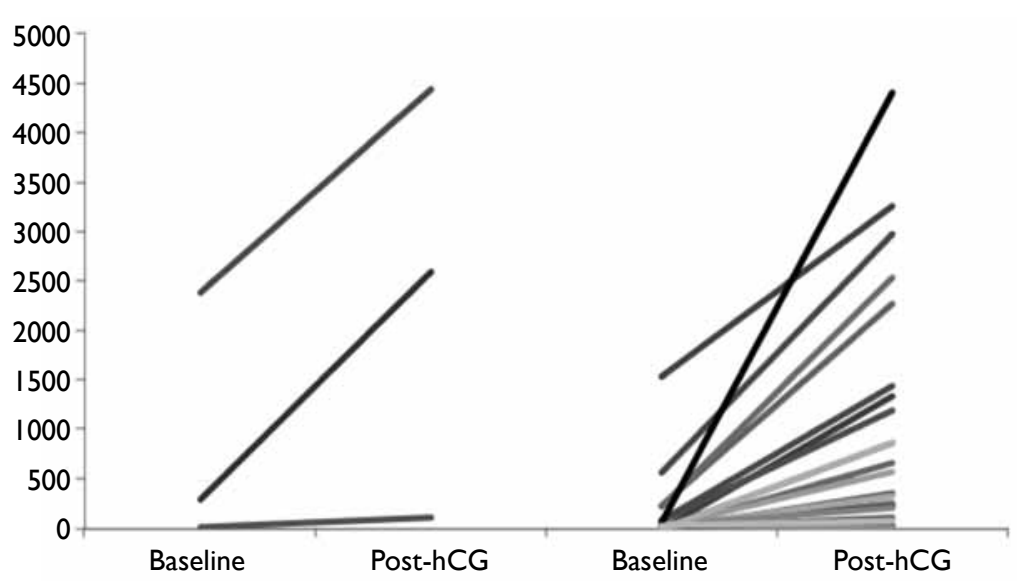

FIG I. Responses in (a) testosterone and (b) dihydrotestosterone levels upon hCG stimulation in patients with $A R$ mutation (left panel) compared with those without (right panel)

Abbreviation: hCG = human chorionic gonadotropin that $5 \mathrm{ARD}$ is excluded in all $46, \mathrm{XY}$ DSD patients before other differential diagnoses are considered. Moreover, since the baseline and post-hCGstimulated $\mathrm{T}$ and DHT results are unreliable when diagnosing AIS, genetic study of the $A R$ gene should also be performed as a first-line investigation.

$H S D 17 B 3$ deficiency has been reported to be the most common cause of $\mathrm{T}$ biosynthetic defect leading to 46,XY DSD in some populations, with an estimated incidence of 1:147 000 in the Netherlands and as high as 1:200 to 1:300 in Arabians due to their high consanguinity rate. ${ }^{16,17}$ Nonetheless, no patient in our cohort was diagnosed with this condition based on the hormonal pattern. Ethnic differences in disease spectrum may be one of the reasons for this observation. Another possible explanation is the lack of reliable diagnostic cutoff for the pre- and post-stimulated T/A4 ratios. George et $\mathrm{a}^{18}$ have summarised the cutoffs used by various researchers, with the pre-stimulated cutoff range set at 0.006 to 1.64 , and the post-stimulated level set at 0.09 to 3.4 for newborn to teenage groups. The difficulties in setting up reliable diagnostic cutoffs for the T/A4 ratio are similar to the T/DHT ratios and have been discussed in our previous study. ${ }^{14}$ Furthermore, $H S D 17 B 3$ deficiency gives no characteristic findings on urinary steroid profiling. ${ }^{5,19}$ Molecular analysis of the HSD17B3 gene may have offered a means to diagnose this condition but unfortunately, due to budget constraints, we were unable to perform mutational analysis of this gene in all our patients, although a normal MLPA result in our patients made gross deletion in this gene unlikely.

The two novel mutations p.Asp266Asn and p.Thr576Pro in the $A R$ gene lie within the Nterminal domain of the androgen receptor that is involved in transcription regulation and DNA binding, respectively. Missense mutations around these two codons have been reported in patients with AIS according to the Androgen Receptor Gene Mutations Database, April 2013. ${ }^{20}$ Multiple sequence alignment shows that both amino acids are highly conserved among different species, suggesting that aspartic acid at codon 266 and threonine at codon 576 are critical for proper receptor function. Similarly, the alanine at codon 260 of the NR5A1 gene is located in helix 3 of the ligand-binding domain of the nuclear receptor, ${ }^{21}$ and is also a highly conserved region. Mutation in this region has been reported to result in $46, \mathrm{XY}$ DSD. ${ }^{8}$ Replacing alanine at this position by valine is therefore expected to be deleterious to the protein function. Phenotypic variability in NR5A1 gene mutation within a kindred has been reported and this may explain why patient 21 had ambiguous external genitalia to such an extent that he required the attention of a paediatric specialist, even though his father was fertile, and denied any symptoms of DSD or need for medical 
TABLE 3. Genetic findings of patients in this study

\begin{tabular}{|c|c|c|}
\hline Patient No. & Gene & Genetic findings \\
\hline 1 & SRD5A2 & Compound heterozygous p.Gln6*/p.Arg227Gln \\
\hline $2^{*}$ & SRD5A2 & Homozygous p.Gly203Ser \\
\hline $3^{*}$ & SRD5A2 & Homozygous p.Arg227Gln \\
\hline $4^{\star}$ & SRD5A2 & Compound heterozygous c.548-1G>A/p.Ala228Val \\
\hline 5 & SRD5A2 & Compound heterozygous p.Leu55Pro/p.Arg227Gln \\
\hline 6 & SRD5A2 & Compound heterozygous p.Val10Gly? p.Arg227Gln \\
\hline $7^{*}$ & SRD5A2 & Homozygous p.Arg246Gln \\
\hline $8^{\star}$ & SRD5A2 & Homozygous p.Arg227Gln \\
\hline $9^{*}$ & SRD5A2 & Homozygous p.Arg227Gln \\
\hline 10 & SRD5A2 & Compound heterozygous p.Gln6*/p.Arg227Gln \\
\hline 11 & SRD5A2 & Homozygous p.Arg227Gln \\
\hline $12 \dagger$ & $A R$ & Hemizygous p.Thr576Pro, mosaic \\
\hline 13 & $A R$ & Hemizygous p.Asp266Asn \\
\hline $14^{*}$ & $A R$ & Hemizygous p.Ala871Val \\
\hline $15 \dagger$ & $A R$ & Hemizygous p.Met788Val, mosaic \\
\hline 16 & $A R$ & Hemizygous p.Ser176Arg \\
\hline 17 & $A R$ & Hemizygous p.Gln825Lys \\
\hline 18 & $A R$ & Hemizygous p.Arg841His \\
\hline 19 & CYP11A1 & Compound heterozygous p.Arg360Trp /p.Arg405* \\
\hline $20 \dagger$ & WT1 & Heterozygous c. $1228+4 \mathrm{C}>\mathrm{T}$ \\
\hline 21 & NR5A1 & Heterozygous p.Ala260Val \\
\hline 22 & $A M H$ & Compound heterozygous p.Cys492Arg/p.Ala546Thr/p.His547Asp \\
\hline 30 & $A M H, W T 1$ & Wild-type sequence \\
\hline 40 & $A M H$ & Wild-type sequence \\
\hline
\end{tabular}

* Genotyping was not performed on the parents

+ De-novo mutation

TABLE 4. In-silico analysis of the novel variants detected in patients with 46,XY disorders of sex development

\begin{tabular}{llccc}
\hline Gene & Novel variant & SIFT & PolyPhen2 & Align GVGD \\
\hline$A M H$ & p.Cys492Arg & Not tolerated & Probably damaging & Class 65 \\
$A M H$ & p.Ala546Thr & Not tolerated & Probably damaging & Class 55 \\
$A M H$ & p.His547Asp & Tolerated & Probably damaging & Class 65 \\
$A R$ & p.Asp266Asn & Not tolerated & Probably damaging & Class 15 \\
$A R$ & p.Thr576Pro & Not tolerated & Probably damaging & Class 35 \\
NR5A1 & p.Ala260Val & Not tolerated & Benign & Class 65 \\
\hline
\end{tabular}

Abbreviation: SIFT = sorting intolerant from tolerant

attention. ${ }^{22}$ For the $A M H$ gene, the 3 ' end of exon 5 abnormal protein folding and increased instability, is one of the mutational hotspots in patients with as reported previously in mutations located in this PMDS. ${ }^{23}$ Exon 5 encodes the bioactive C-terminal region. ${ }^{24}$

domain. The three mutations detected in patient 22 Gonadal malignancy was rare in our series, are all located at highly conserved regions. Although probably because gonadectomy was performed early in-vitro functional characterisation for the mutant in life when the decision of female sex assignment proteins was not performed, the undetectable serum was made. Although this helps to avoid further AMH level in this patient was compatible with virilisation and to establish gender identity, the the mutations being pathogenic, possibly due to timing of corrective surgery and gonadectomy 
(a)

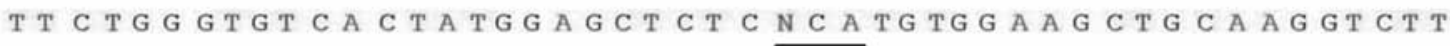

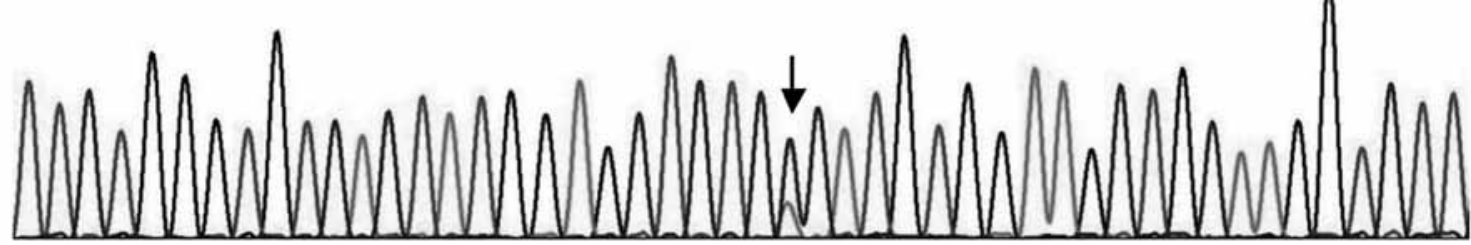

(b)

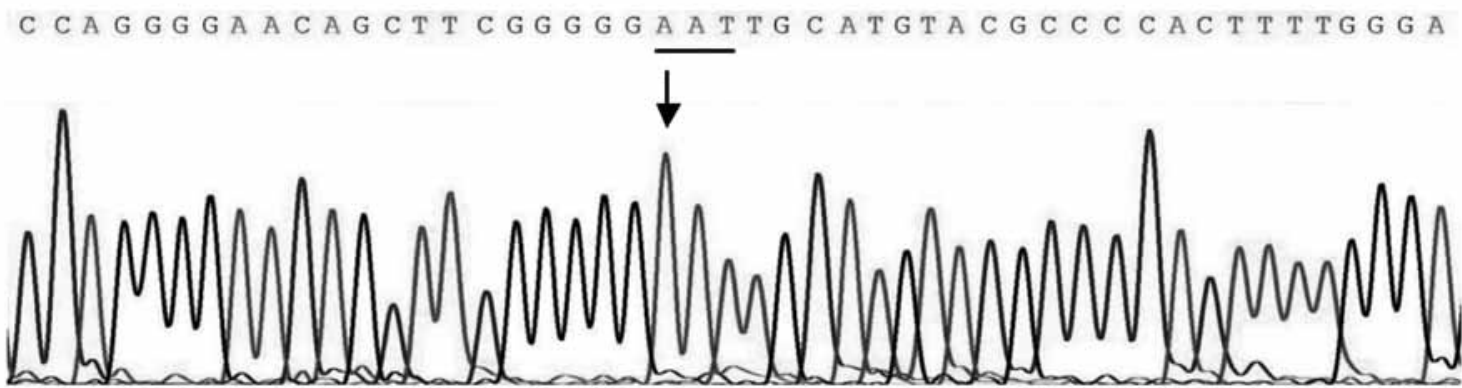

(c)

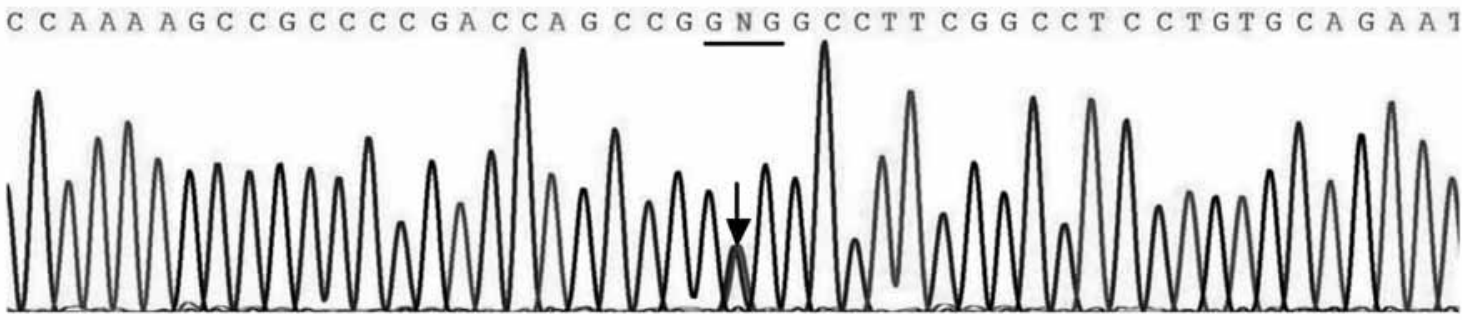

(d)

C CA A C A A T T C CA G G C G T G G C G G C T G G C T CA T C C GAC C G C A A C

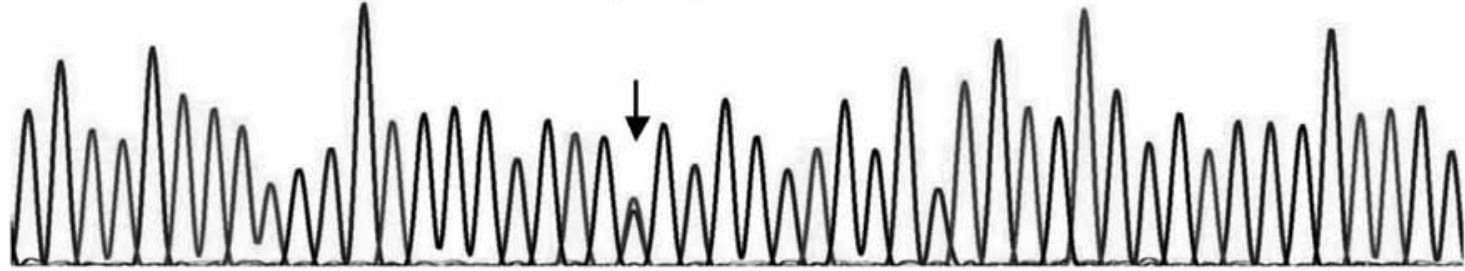

(e)

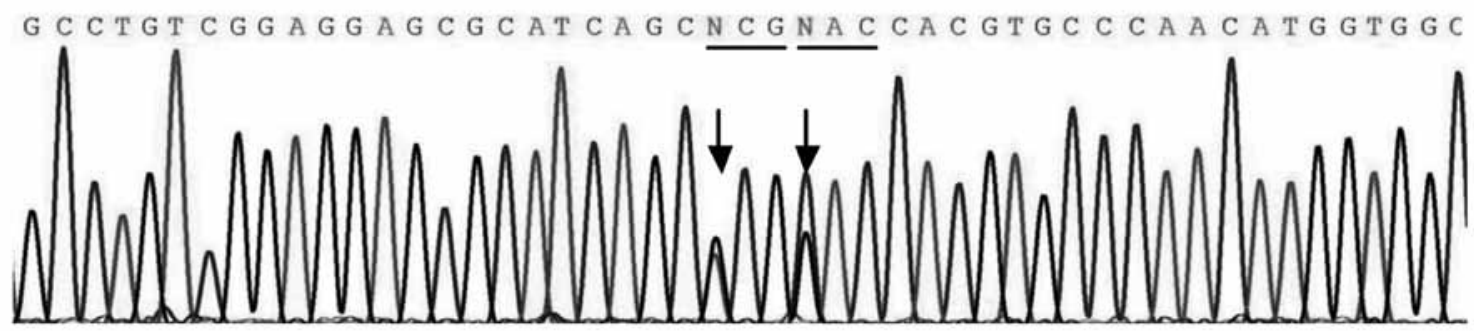

FIG 2. Segments of electropherograms showing the novel mutations

(a) Hemizygous c. 1726A>C, p.Thr576Pro in mosaic pattern in the AR gene in patient 12; (b) hemizygous c.796G >A, p.Asp266Asn in the AR gene in patient I3; (c) heterozygous c.779C >T, p.Ala260Val in the NR5A I gene in patient 21; (d) heterozygous c. I 474T>C, p.Cys492Arg; and (e) heterozygous c. 1636G>A, p.Ala546Thr and c. 1639C C G, p. His547Asp in the AMH gene in patient 22. The heterozygous sites are denoted by the letter $\mathrm{N}$ and the mutation site is indicated by arrows. The mutated codon is underlined 
remain controversial. Patient advocacy groups have suggested delaying any surgery for cosmetic reasons until the patient is mature enough to give informed consent ${ }^{25}$ but such practice has not been validated in our Chinese patients. Whether cultural factors have any impact on gender assignment remains uncertain in our community.

Prematurity or low birth weight was not uncommon in our series. This made diagnosis of DSD in our patients even more difficult because ethnic-specific and gestational age- or weightadjusted anthropometric measurement of the external genitalia was not available. Assessment of the genital anatomy relies solely on the experience of the paediatric specialist and is obviously far from ideal. A conjoint effort by local paediatricians is needed to set up these normative data.

Less than half of our patients had a confirmed diagnosis in the present study. With the increasing availability of next-generation sequencing technology, and with its established role in molecular diagnostic services, including DSD, ${ }^{3,26}$ it is hoped that sooner rather than later, most patients will have a confirmed genetic diagnosis. Nonetheless, we speculate that some patients have a non-genetic aetiology since environmental factors may alter the phenotypic expression. Several animal and human studies have shown that antenatal exposure to pesticides and plasticisers may lead to fetal genital malformation. ${ }^{27}$

Altogether there was an average of 11250 male live births every year in the five public hospitals that participated in this study. Since 11 newborns with 46,XY DSD were born in these five hospitals and were recruited during our study period, this gives an estimated incidence of 46,XY DSD of 1:2045 male births requiring the input of paediatric endocrinologists. This figure may underestimate the true incidence of this group of diseases as some patients present late and others may have subtle defects that go unnoticed by our specialists. If the actual number of patients with chromosomal and 46 ,XX DSD in our population is considered, the actual incidence of DSD can be expected to be much higher.

There are a few limitations in this study. First, the number of patients was relatively small. This may have resulted in bias in our observation and the data do not represent the prevalence of disease in our population. Second, in-vitro study was not performed on the novel genetic variants for functional characterisation, although we believe that all the available evidence indicates the pathogenic nature of these variants. Third, due to budget constraints, we were unable to sequence all genes related to $46, \mathrm{XY}$ DSD.

\section{Conclusions}

Our findings indicate that 5ARD and AIS are possibly the major causes of 46,XY DSD in the Hong Kong Chinese population. Molecular analyses of the SRD5A2 and $A R$ genes were demonstrated to be more reliable than hormonal testing. Since the missense mutation p.Arg227Gln was a recurrent hotspot mutation in 5ARD in our local patients, all patients should be screened for this mutation.

\section{Acknowledgements}

We thank Mr YC Ho, Ms YF Wong, and Ms YP Iu for their technical assistance. The study was supported by the Queen Elizabeth Hospital Research Grant 2009 QEH/RC/G/0910-A04/R0901 and Kowloon Central Cluster Research Grant 2012 KCC/RC/ G/1213-B01.

\section{References}

1. Lee PA, Houk CP, Ahmed SF, Hughes IA; International Consensus Conference on Intersex organized by the Lawson Wilkins Pediatric Endocrine Society and the European Society for Paediatric Endocrinology. Consensus statement on management of intersex disorders. International Consensus Conference on Intersex. Pediatrics 2006;118.e488-500.

2. Ono M, Harley VR. Disorders of sex development: new genes, new concepts. Nat Rev Endocrinol 2013;9:79-91.

3. Arboleda VA, Lee H, Sánchez FJ, et al. Targeted massively parallel sequencing provides comprehensive genetic diagnosis for patients with disorders of sex development. Clin Genet 2013;83:35-43.

4. Jääskeläinen J. Molecular biology of androgen insensitivity. Mol Cell Endocrinol 2012;35:4-12.

5. Chan AO, Shek CC. Urinary steroid profiling in the diagnosis of congenital adrenal hyperplasia and disorders of sex development: experience of a urinary steroid referral centre in Hong Kong. Clin Biochem 2013;46:327-34.

6. Parajes S, Chan AO, But WM, et al. Delayed diagnosis of adrenal insufficiency in a patient with severe penoscrotal hypospadias due to two novel $\mathrm{P} 450$ side-chain cleavage enzyme (CYP11A1) mutations (p.R360W; p.R405X). Eur J Endocrinol 2012;167:881-5.

7. Chan WK, To KF, But WM, Lee KW. Frasier syndrome: a rare cause of delayed puberty. Hong Kong Med J 2006;12:225-7.

8. Allali S, Muller JB, Brauner R, et al. Mutation analysis of NR5A1 encoding steroidogenic factor 1 in 77 patients with 46,XY disorders of sex development (DSD) including hypospadias. PLoS One 2011;6:e24117.

9. Bangsbøll S, Qvist I, Lebech PE, Lewinsky M. Testicular feminization syndrome and associated gonadal tumors in Denmark. Acta Obstet Gynecol Scand 1992;71:63-6.

10. Boehmer AL, Brinkmann $\mathrm{O}$, Brüggenwirth $\mathrm{H}$, et al. Genotype versus phenotype in families with androgen insensitivity syndrome. J Clin Endocrinol Metab 2001;86:4151-60.

11. Abdullah MA, Saeed U, Abass A, et al. Disorders of sex development among Sudanese children: 5-year experience of a pediatric endocrinology clinic. J Pediatr Endocrinol Metab 2012;25:1065-72.

12. Mieszczak J, Houk CP, Lee PA. Assignment of the sex of rearing in the neonate with a disorder of sex development. 
Curr Opin Pediatr 2009;21:541-7.

13. Imperato-McGinley J, Peterson RE, Gautier T, Sturla E. Androgens and the evolution of male-gender identity among male pseudohermaphrodites with 5alpha-reductase deficiency. N Engl J Med 1979;300:1233-7.

14. Chan AO, But BW, Lee CY, et al. Diagnosis of $5 \alpha$-reductase 2 deficiency: is measurement of dihydrotestosterone essential? Clin Chem 2013;59:798-806.

15. Chan AO, But BW, Lau GT, et al. Diagnosis of $5 \alpha$-reductase 2 deficiency: a local experience. Hong Kong Med 2009;15:130-5.

16. Boehmer AL, Brinkmann AO, Sandkuijl LA, et al. 17 $\beta$ hydroxysteroid dehydrogenase-3 deficiency: diagnosis, phenotypic variability, population genetics, and worldwide distribution of ancient and de novo mutations. J Clin Endocrinol Metab 1999;84:4713-21.

17. Rosler A. 17 Beta-hydroxysteroid dehydrogenase 3 deficiency in the Mediterranean population. Pediatr Endocrinol Rev 2006;3 Suppl 3:455-61.

18. George MM, New MI, Ten S, Sultan C, Bhangoo A. The clinical and molecular heterogeneity of $17 \beta \mathrm{HSD}-3$ enzyme deficiency. Horm Res Paediatr 2010;74:229-40.

19. Lee YS, Kirk JM, Stanhope RG, et al. Phenotypic variability in $17 \beta$-hydroxysteroid dehydrogenase-3 deficiency and diagnostic pitfalls. Clin Endocrinol 2007;67:20-8.

20. Androgen Receptor Gene Mutations Database. Available from: http://androgendb.mcgill.ca/. Accessed Aug 2013.

21. El-Khairi R, Martinez-Aguayo A, Ferraz-de-Souza B, Lin L, Achermann JC. Role of DAX-1 (NROB1) and steroidogenic factor-1 (NR5A1) in human adrenal function. Endocr Dev
2011:20:38-46

22. Ciaccio M, Costanzo M, Guercio G, et al. Preserved fertility in a patient with a $46, \mathrm{XY}$ disorder of sex development due to a new heterozygous mutation in the NR5A1/SF-1 gene: evidence of $46, \mathrm{XY}$ and $46, \mathrm{XX}$ gonadal dysgenesis phenotype variability in multiple members of an affected kindred. Horm Res Paediatr 2012;78:119-26.

23. Josso N, Belville C, di Clemente N, Picard JY. AMH and AMH receptor defects in persistent Müllerian duct syndrome. Hum Reprod Update 2005;11:351-6.

24. Belville C, Van Vlijmen H, Ehrenfels C, et al. Mutations of the anti-Müllerian hormone gene in patients with persistent Müllerian duct syndrome: biosynthesis, secretion, and processing of the abnormal proteins and analysis using a three-dimensional model. Mol Endocrinol 2004;18:708-21.

25. Consortium on the Management of Disorders of Sex Development: Clinical Guidelines for the Management of Disorders of Sex Development in Childhood. California, US: Intersex Society of North America; 2006. Available from: http://www.dsdguidelines.org/files/clinical.pdf. Accessed Aug 2015.

26. Hersmus R, Stoop H, Turbitt E, et al. SRY mutation analysis by next generation (deep) sequencing in a cohort of chromosomal Disorders of Sex Development (DSD) patients with a mosaic karyotype. BMC Med Genet 2012;13:108.

27. Kalfa N, Philibert PH, Baskin LS, Sultan C. Hypospadias: interactions between environment and genetics. Mol Cell Endocrinol 2011;335:89-95. 